\title{
Intervenções vocais diretas e indiretas em professores: revisão sistemática da literatura
}

\section{Direct and indirect vocal interventions on teachers: systematic review of the literature}

\author{
Tanise Cristaldo Anhaia', Léia Gonçalves Gurgel², Raquel Hochmuller Vieira³, Mauriceia Cassol${ }^{4}$
}

\begin{abstract}
RESUMO
Objetivo: Verificar a eficácia das intervenções vocais diretas e indiretas, de forma isolada e combinada, na prevenção de distúrbios vocais em professores, por meio de revisão sistemática da literatura. Estratégias de pesquisa: Foram pesquisados artigos de janeiro de 1980 a abril de 2013, nas bases de dados eletrônicas MEDLINE (acessado pelo PubMed), PubMed, LILACS, SciELO, Scopus e Web of Science. Critérios de seleção: Foram incluídos todos os artigos que tinham como objetivo principal algum tipo de intervenção com professores e que fossem ensaios controlados randomizados. Excluíram-se artigos que apresentavam indivíduos com alterações de laringe e voz e que estivessem afastados de sua profissão. Resultados: Como resultado da busca inicial, foram identificados 677 estudos, dentre os quais, cinco atendiam aos critérios de inclusão. Somou-se a esses mais quatro artigos, encontrados nas referências bibliográficas dos estudos selecionados para a fase de leitura do texto completo. Conclusão: A intervenção combinada (direta e indireta) apresentou melhora significativa em parâmetros da qualidade vocal e na autoavaliação da voz, mesmo em curto período de tempo. Em outros estudos, cujo foco era a comparação entre a intervenção combinada e intervenções isoladas (direta ou indireta), não foram observadas diferenças significativas, apesar de serem descritas melhoras em alguns dos parâmetros vocais avaliados. Como limitação desta revisão, pode-se incluir a restrição quanto ao desenho metodológico dos estudos, incluindo apenas ensaios clínicos randomizados. A intervenção vocal combinada apresenta resultados mais expressivos do que a intervenção isolada.
\end{abstract}

Descritores: Voz; Docentes; Treinamento da Voz; Distúrbios da Voz; Laringe; Saúde do Trabalhador

\begin{abstract}
Purpose: The aim of this work is to verify the efficacy of direct and indirect vocal interventions in preventing voice disorders, with specific and combined strategies, by a systematic review of the literature. Research strategies: Articles published from January, 1980 to April, 2013 were searched in the electronic databases MEDLINE (accessed through PubMed), PubMed, LILACS, SciELO, Scopus and Web of Science. Selection criteria: All the articles that presented randomized controlled studies with some type of vocal intervention with teachers as their primary aim were included. Articles that presented subjects with larynx and voice alterations were excluded. Results: As a result of the initial search, 677 studies were identified, five of which followed the inclusion criteria. Four more articles, found in the references of the studies selected for reading of the full text, were included. Conclusion: The combined intervention (direct and indirect) presented a significant improvement in vocal quality parameters and self-assessment, even in a short period of time. In other studies, which focused on the comparison between combined and specific interventions (direct or indirect), no differences were observed, although improvements in some of the assessed vocal parameters were described. A limitation of this review is the restriction of the methodological design of the studies, including only randomized clinical trials. The combined vocal intervention presented more significant results than the specific intervention.
\end{abstract}

Keywords: Voice; Faculty; Voice Training; Voice Disorders; Larynx; Occupational Health

Trabalho realizado na Universidade Federal de Ciências da Saúde de Porto Alegre - UFCSPA - Porto Alegre (RS), Brasil.

(1) Programa de Pós-graduação (Mestrado) em Ciências da Reabilitação, Universidade Federal de Ciências da Saúde de Porto Alegre - UFCSPA - Porto Alegre (RS), Brasil.

(2) Programa de Pós-graduação (Mestrado) em Ciências da Saúde, Universidade Federal de Ciências da Saúde de Porto Alegre - UFCSPA - Porto Alegre (RS), Brasil. (3) Curso de Fonoaudiologia, Universidade Federal de Ciências da Saúde de Porto Alegre - UFCSPA - Porto Alegre (RS), Brasil.

(4) Programa de Pós-graduação em Ciências da Reabilitação, Universidade Federal de Ciências da Saúde de Porto Alegre - UFCSPA - Porto Alegre (RS), Brasil. Conflito de interesses: Não

Contribuição dos autores: TCA: pesquisadora principal, elaboração da pesquisa, elaboração do cronograma, levantamento da literatura, coleta e análise dos dados, redação do artigo, submissão e trâmites do artigo; $L G G$ : elaboração da pesquisa, elaboração do cronograma, levantamento da literatura, coleta e análise dos dados, redação do artigo, submissão e trâmites do artigo; $R H V$ : elaboração da pesquisa, elaboração do cronograma, levantamento da literatura, coleta e análise dos dados, redação do artigo, submissão e trâmites do artigo; $M C$ : orientadora, correção da redação do artigo, aprovação da versão final.

Endereço para correspondência: Tanise Cristaldo Anhaia. R. Riachuelo 1290/1004, Porto Alegre (RS), Brasil, CEP: 90010-270.

E-mail: tanisecristaldo@ hotmail.com

Recebido em: 29/4/2013; Aceito em: 4/9/2013 


\section{INTRODUÇÃO}

Considerada fator relevante para o processo de socialização humana, a voz produz impactos na qualidade de vida dos indivíduos, especialmente daqueles que a utilizam profissionalmente ${ }^{(1)}$. Em razão disso, pesquisas envolvendo o uso profissional da voz têm recebido maior atenção nos últimos anos ${ }^{(2,3)}$. Sabese que os professores apresentam maior risco de desenvolver alterações vocais, quando comparados a sujeitos da população em geral $^{(4)}$, e, pelo menos $50 \%$ deles sofrem, regularmente, com distúrbios de voz, limitando seu desempenho no trabalho ${ }^{(5)}$.

O objetivo dos cuidados vocais, tanto de prevenção, quanto de tratamento, é recuperar a voz, tornando-a funcional para o uso profissional e para a comunicação, em geral. Diante disso, as intervenções destinadas a prevenir os distúrbios da voz podem ser divididas em intervenções diretas e indiretas ${ }^{(6)}$. A abordagem direta proporciona a mudança do funcionamento vocal, oferecendo instruções de técnicas para a voz, a fim de incentivar a produção mais eficaz e, assim, proteger o indivíduo quanto ao desenvolvimento de problemas de voz. Por outro lado, a abordagem indireta auxilia o indivíduo a compreender o uso vocal, os fatores psicológicos e ambientais que podem levar a alteração de voz e a desenvolver estratégias que minimizem tais fatores de risco $^{(4)}$.

$\mathrm{O}$ treinamento e a terapia vocal objetivam a prevenção e o tratamento de distúrbios da voz, através da mudança dos hábitos vocais. O treinamento vocal torna o uso da voz mais fácil e suave, evita quadros de fadiga vocal e prática inadequada e proporciona melhor preparação para o desempenho da atividade que o requer ${ }^{(7)}$. Além disso, fisiologicamente, os exercícios de treinamento vocal têm a função de melhorar a circulação sanguínea e a respiração, permitindo o aumento das contrações e elasticidade musculares ${ }^{(8)}$. Vários estudos têm demonstrado efeitos positivos da terapia e do treinamento vocal, em relação aos parâmetros avaliados nas análises acústica e perceptivo-auditiva, em termos da diminuição da perturbação (jitter e shimmer), aumento do sinal-ruído, além de melhor qualidade $\operatorname{vocal}^{(9)}$. No entanto, poucos programas de prevenção são projetados para professores e futuros professores ${ }^{(7)}$.

Considerando que a perspectiva da avaliação vocal tem sido privilegiada nas pesquisas brasileiras ${ }^{(10)}$, os estudos sobre efeitos de intervenções na voz desses profissionais são mais recentes e auxiliam na compreensão da complexa realidade do uso da voz na docência e a nortear futuras ações fonoaudiológicas. Os estudos de revisão sistemática têm reconhecida importância na área da saúde e, embora não frequentes na Fonoaudiologia, vêm aumentando na última década, enfatizando a necessidade de reflexões em torno das temáticas utilizadas e identificação de novos rumos, a partir das análises realizadas.

\section{OBJETIVOS}

Diante do exposto, o objetivo deste estudo foi verificar a eficácia das intervenções vocais diretas e indiretas, de forma isolada e combinada, na prevenção de distúrbios vocais em professores.

\section{ESTRATÉGIA DE PESQUISA}

Foram pesquisadas as seguintes bases de dados eletrônicas, de janeiro de 1980 a abril de 2013: MEDLINE (acessado pelo PubMed), PubMed, LILACS, SciELO, Scopus e Web of Science. Os termos de busca utilizados foram voice, faculty e randomized controlled trial e seus entretermos, em Português, Inglês e Espanhol. Não foram incluídas palavras relacionadas aos desfechos de interesse, a fim de que a sensibilidade desta pesquisa fosse ampliada. Não houve restrição quanto ao tipo de intervenção utilizada nos estudos.

\section{CRITÉRIOS DE SELEÇÃO}

Foram incluídos todos os ensaios controlados randomizados que apresentavam, como objetivo principal, a realização de algum tipo de intervenção vocal com professores. Optou-se pela restrição do delineamento, pois os ensaios clínicos randomizados são a fonte de evidência científica mais confiável, fornecendo maior validade aos resultados de uma revisão sistemática. Os critérios de exclusão para esta revisão foram: estudos que incluíam indivíduos que apresentavam alterações prévias de laringe e de voz e estudos que incluíam professores que não exerciam mais sua atividade profissional ou que estivessem afastados por algum motivo. Não houve limite de idade, nem restrição de gênero e de tempo de intervenção.

\section{ANÁLISE DOS DADOS}

Na primeira fase, os títulos e os resumos de todos os artigos identificados pela estratégia de busca foram avaliados pelos investigadores. $\mathrm{Na}$ fase seguinte, todos os resumos que não forneceram informações suficientes em relação aos critérios de inclusão e exclusão e que continham informações sobre intervenções com professores, foram selecionados para avaliação do texto integral. Na terceira fase, avaliação do texto completo dos estudos, dois revisores, previamente treinados, e com formulários padronizados de preenchimento, avaliaram os artigos completos, independentemente, e realizaram suas seleções de acordo com os critérios predeterminados de elegibilidade dos estudos. A seção de referências bibliográficas desses artigos também foi revisada, buscando-se encontrar trabalhos que, por algum motivo, não tivessem aparecido na busca realizada. As discordâncias, em todas as fases de seleção dos estudos, foram resolvidas por consenso. Os dados primários foram coletados, a respeito do método de intervenção vocal em professores.

A qualidade dos estudos foi avaliada através do Manual Cochrane ${ }^{(11)}$, que classifica os estudos em A, B ou C, de acordo com a baixa, moderada ou alta chance de viés dos estudos primários, respectivamente. Tal classificação se detém na validade 
interna dos estudos, na sua forma de aleatorização e no modo como contornou ou minimizou os vieses. A avaliação da qualidade dos estudos foi complementada com a utilização da escala $\mathrm{Jadad}^{(12)}$, um instrumento de avaliação da qualidade, que tem cinco questões envolvendo a randomização, mascaramento, duplo-cegos, perdas e exclusões.

\section{RESULTADOS}

Como resultado da busca inicial, foram identificados 677 estudos, dentre os quais, cinco ${ }^{(5,9,13-15)}$ atendiam aos critérios de inclusão e foram considerados relevantes para a amostra deste trabalho. A esses, foram somados mais quatro artigos ${ }^{(3,4,16,17)}$, classificados como referências adicionais, encontrados nas referências bibliográficas dos estudos selecionados para a fase de leitura do texto completo. Portanto, foram incluídos nove artigos na presente revisão, tendo sido analisados com maior rigor (Figura 1).

Os artigos, em sua totalidade, foram publicados em língua inglesa e 44,4\% dos estudos incluídos foram realizados na Finlândia. Quinhentos e quarenta e três indivíduos, com média de idade entre 24 e 42 anos, foram incluídos nos nove estudos. Alguns estudos ${ }^{(3,9,13,16)}$, com qualidade que variou de fraca a boa, incluíram apenas participantes do gênero feminino. $\mathrm{O}$ resultado da autoavaliação vocal foi o parâmetro mais utilizado (em 87,5\% dos estudos incluídos) para análise do efeito das intervenções.

As principais características dos estudos incluídos, como autores, ano de publicação, país de origem, língua original, tipo de intervenção realizada, número de participantes, idade e gênero da amostra estão descritos na Tabela 1.

Em relação à qualidade dos estudos, observou-se que esteve entre fraca e boa, conforme demonstrado na avaliação completa (Tabela 2).

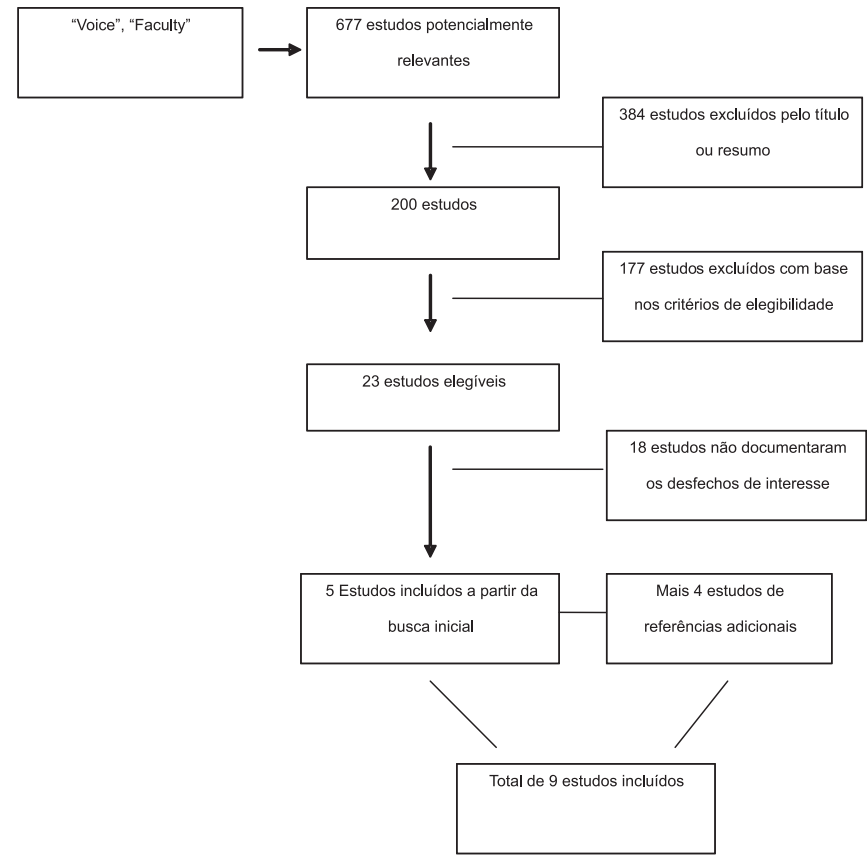

Figura 1. Diagrama de seleção dos artigos

\section{DISCUSSÃo}

Na literatura, alguns estudos clássicos ${ }^{(5,13)}$, orientam a prática de intervenções diretas, ou seja, que se aplicam diretamente ao aparelho fonador, e indiretas como, por exemplo, consultoria ou educação sobre higiene vocal e melhora das condições acústicas do ambiente de trabalho(6).

Quatro estudos incluídos nesta revisão compararam as intervenções vocais direta e indireta ${ }^{(4,5,11,13)}$. Um desses estu$\operatorname{dos}^{(13)}$, verificou o efeito da intervenção vocal na autopercepção de mulheres, após o treinamento vocal direto e indireto. Os

Tabela 1. Características dos estudos incluídos nesta revisão

\begin{tabular}{|c|c|c|c|c|c|c|}
\hline Autores e ano & $\begin{array}{l}\text { País de } \\
\text { origem }\end{array}$ & $\begin{array}{l}\text { Língua } \\
\text { original }\end{array}$ & Periódico (fator de impacto) & $\begin{array}{c}\text { Tipo de } \\
\text { intervenção }\end{array}$ & $\begin{array}{c}\mathrm{n} \text { (média de idade } \\
\text { da amostra) }\end{array}$ & Gênero \\
\hline $\begin{array}{l}\text { Laukkanen et al } \\
\text { (2009) }\end{array}$ & Finlândia & Inglês & $\begin{array}{c}\text { Folia Phoniatrica et } \\
\text { Logopaedica }(0,726)\end{array}$ & Direta e indireta & $\mathrm{n}=90$ (41,1 anos) & $\begin{array}{l}F=90 \\
M=0\end{array}$ \\
\hline $\begin{array}{l}\text { Timmermans et al } \\
\text { (2011) }\end{array}$ & Bélgica & Inglês & Jounal of Voice $(1,108)$ & Direta e Indireta & $\mathrm{n}=65(\mathrm{nc})$ & $\begin{array}{l}F=43 \\
M=22\end{array}$ \\
\hline $\begin{array}{l}\text { Gillivan-Murphy et } \\
\text { al (2006) }\end{array}$ & Irlanda & Inglês & Jounal of Voice $(1,108)$ & Direta e indireta & $\mathrm{n}=20$ (40 anos) & $\begin{array}{l}\mathrm{F}=\mathrm{nc} \\
\mathrm{M}=\mathrm{nc}\end{array}$ \\
\hline $\begin{array}{l}\text { Duffy \& Hazlett } \\
(2004)\end{array}$ & Irlanda & Inglês & Jounal of Voice $(1,108)$ & Direta e indireta & $\begin{array}{c}\mathrm{n}=55 \text { (entre } 24 \text { e } \\
25 \text { anos) }\end{array}$ & $\begin{array}{l}\mathrm{F}=\mathrm{nc} \\
\mathrm{M}=\mathrm{nc}\end{array}$ \\
\hline Bovo et al (2006) & Itália & Inglês & Jounal of Voice $(1,108)$ & Direta e Indireta & $\begin{array}{c}\mathrm{n}=64 \text { (entre } 38 \mathrm{e} \\
39 \text { anos) }\end{array}$ & $\begin{array}{l}\mathrm{F}=\mathrm{nc} \\
\mathrm{M}=\mathrm{nc}\end{array}$ \\
\hline $\begin{array}{l}\text { Leppänen et al } \\
\text { (2009) }\end{array}$ & Finlândia & Inglês & $\begin{array}{l}\text { Folia Phoniatrica et } \\
\text { Logopaedica }(0,726)\end{array}$ & Direta e indireta & $n=60$ (40,6 anos $)$ & $\begin{array}{l}F=60 \\
M=0\end{array}$ \\
\hline $\begin{array}{l}\text { Leppänen et al } \\
\text { (2010) }\end{array}$ & Finlândia & Inglês & $\begin{array}{c}\text { Logopedics Phoniatrics } \\
\text { Vocology }(0,83)\end{array}$ & Direta e indireta & $\mathrm{n}=90$ (41anos) & $\begin{array}{l}F=90 \\
M=0\end{array}$ \\
\hline Pasa et al (2007) & Austrália & Inglês & $\begin{array}{l}\text { Logopedics Phoniatrics } \\
\text { Vocology }(0,83)\end{array}$ & Direta e indireta & $\mathrm{n}=39$ (38 anos) & $\begin{array}{l}F=36 \\
M=3\end{array}$ \\
\hline $\begin{array}{l}\text { Ilomaki et al } \\
(2008)\end{array}$ & Finlândia & Inglês & $\begin{array}{c}\text { Logopedics Phoniatrics } \\
\text { Vocology }(0,83)\end{array}$ & Direta e indireta & $\mathrm{n}=60$ (42 anos) & $\begin{array}{l}F=60 \\
M=0\end{array}$ \\
\hline
\end{tabular}

Legenda: $\mathrm{nc}=$ não consta; $\mathrm{F}=$ feminino; $\mathrm{M}=$ masculino 
Tabela 2. Avaliação de qualidade dos estudos

\begin{tabular}{|c|c|c|c|c|c|}
\hline \multirow[b]{2}{*}{ Estudo (ano) } & \multicolumn{2}{|c|}{ Aleatorização } & \multirow[b]{2}{*}{ Mascaramento } & \multirow[b]{2}{*}{$\begin{array}{l}\text { Perdas no } \\
\text { seguimento }\end{array}$} & \multirow[b]{2}{*}{ Jadad (1996) } \\
\hline & $\begin{array}{c}\text { Sequência de } \\
\text { alocação }\end{array}$ & $\begin{array}{l}\text { Sigilo de } \\
\text { alocação }\end{array}$ & & & \\
\hline Duffy e Hazlett (2004) & $\mathrm{A}$ & B & $\mathrm{B}$ & $\mathrm{B}$ & 3 \\
\hline Bovo et al (2006) & A & B & C & A & 3 \\
\hline Leppanen et al (2009) & A & B & C & C & $2^{*}$ \\
\hline Laukkanen et al (2009) & A & B & B & C & $2^{*}$ \\
\hline Timmermans et al (2011) & A & B & C & B & 3 \\
\hline Gillivan-Murphy et al (2006) & A & C & B & B & 3 \\
\hline Leppanen et al (2010) & A & B & B & C & $2^{*}$ \\
\hline Pasa et al (2007) & A & B & $\mathrm{B}$ & A & 3 \\
\hline Ilomaki et al (2008) & A & B & B & A & 3 \\
\hline
\end{tabular}

*classificação fraca de acordo com a Escala Jadad (1996)

Legenda: $\mathrm{A}=$ descrição adequada; $\mathrm{B}=$ não descrita; $\mathrm{C}=$ descrição inadequada

autores observaram que ambas as intervenções apresentaram efeitos positivos. No entanto, não foram eficazes para melhorar a autopercepção do funcionamento vocal dos participantes. Diante disso, sugeriu-se que seria interessante investigar os efeitos do aumento do tempo utilizado para os treinamentos, para o conhecimento dos sujeitos participantes da pesquisa, no que diz respeito aos cuidados com a voz.

Outra pesquisa ${ }^{(4)}$, investigou os efeitos das orientações em relação à higiene vocal e da realização de exercícios, propriamente ditos, na redução dos sintomas vocais em professores da escola primária. Os participantes do grupo que recebeu orientações sobre higiene vocal e do grupo que realizou os exercícios de função vocal relataram melhora nas características vocais e maior conhecimento vocal após a intervenção. O grupo controle, no entanto, apresentou dificuldades persistentes nos parâmetros de conhecimento vocal, comportamento vocal no trabalho, sintomas vocais e tempo máximo de fonação. A intervenção indireta foi significativamente mais benéfica, quando comparada com a intervenção direta. Entretanto, esses resultados não concordam com os achados da literatura ${ }^{(5)}$, que concluiu que as intervenções de higiene vocal não são tão eficazes quanto os programas de treinamento vocal, com ou sem orientações sobre cuidados com a voz.

Pesquisa ${ }^{(17)}$ realizada na Finlândia, concluiu que a realização de exercícios vocais é mais efetiva do que a realização isolada de instruções de higiene vocal. Em seu estudo, o grupo que realizou exercícios vocais apresentou redução na frequência fundamental e nas médias de jitter e shimmer, além de maior facilidade de fonação e aperfeiçoamento da qualidade vocal. Em contraponto, o grupo que recebeu orientações sobre educação e higiene vocal apresentou frequência fundamental mais elevada e maior dificuldade de fonação. Esse resultado pode ser justificado pelo fato da higiene vocal tratar apenas de orientações quanto aos cuidados com a voz, ou seja, os efeitos desse tipo de intervenção dependem da absorção das informações, pelo sujeito, e de colocá-las em prática, durante o uso profissional da voz.

Outros estudos incluídos na presente revisão, verificaram os efeitos das intervenções diretas e indiretas combinadas. Em estudo $^{(14)}$ que combinou essas duas abordagens, não foram encontradas diferenças clinicamente significativas entre o grupo experimental e o grupo controle, com relação à avaliação perceptivo-auditiva. No entanto, para os parâmetros de qualidade vocal, que foram mensurados por meio do Índice de Severidade $\mathrm{Vocal}^{(18)}$, várias diferenças significativas foram verificadas. Os indivíduos do grupo de treinamento vocal foram capazes de ampliar seu alcance de voz e alterar o comportamento vocal. Os autores concluíram que, por menor que seja o treinamento vocal combinado, deverá apresentar impacto positivo sobre a voz dos professores. Entretanto, não ficou evidente até que ponto um programa de treinamento mais extenso poderá mostrar resultados promissores.

Um estudo ${ }^{(15)}$ indicou que a combinação do tratamento vocal direto e indireto resulta em melhores relatos dos indivíduos sobre seus sintomas vocais e sobre os cuidados com a voz, além de significativa melhora dos sintomas vocais e dos conhecimentos sobre voz, principalmente quando o terapeuta trabalha com um grupo pequeno de sujeitos. Os autores também sugerem que as qualidades individuais do terapeuta podem ser uma variável determinante na efetividade do tratamento.

Em uma pesquisa ${ }^{(5)}$, a eficácia do treinamento vocal combinado foi comprovada, embora o efeito positivo tenha se reduzido um pouco, no seguimento realizado após um ano. $\mathrm{O}$ grupo experimental apresentou melhora em aspectos como disfonia global, aumento dos tempos máximos de fonação, jitter e shimmer. Não houve diferença significativa para energia de ruído glótico e frequência fundamental. Os autores salientam que a melhora na acústica das salas de aula, a utilização de aparelhos de amplificação durante a atividade profissional e a realização de programas de orientação para professores sobre cuidados vocais, mostram-se como aspectos essenciais para a prevenção primária dos problemas vocais em professores.

Outros estudos ${ }^{(3,9,16)}$, tiveram como objetivo comparar a intervenção vocal indireta em relação à intervenção direta e indireta combinadas. Na primeira pesquisa supracitada ${ }^{(3)}$, os grupos que receberam massagem vocal, treinamento vocal 
e palestras sobre higiene vocal não diferiram, em relação ao seguimento realizado após seis meses e após 12 meses às intervenções. O grupo dos pacientes que recebeu apenas orientações apresentou resultados satisfatórios, em relação ao aprendizado sobre higiene vocal. O grupo que recebeu treinamento vocal, em um primeiro momento, salientou a importância da produção de voz adequada e repouso vocal e, após 12 meses, enfatizou a importância de relaxamento e descanso vocal. O grupo que recebeu massagem vocal nos primeiros seis meses relatou a relevância do repouso vocal, consciência corporal e relaxamento e, após 12 meses, salientou a importância do uso vocal adequado. Em todos os grupos, pôde-se observar que os sintomas de fadiga vocal diminuíram após um ano de acompanhamento, demonstrando que, em diferentes graus, todas as intervenções são válidas para a melhora do bem-estar vocal dos professores.

Em outra pesquisa ${ }^{(9)}$, foram relatados efeitos positivos após todas as intervenções, porém mais significativamente depois do treinamento vocal e da massagem vocal, do que após a palestra sobre higiene vocal. O resultado da autoavaliação, antes e depois de um dia de trabalho, não foi capaz de mostrar efeito significativo após as intervenções.

Um estudo revelou que ${ }^{(16)}$ o grupo que recebeu orientações quanto à higiene vocal e o grupo que recebeu massagem vocal não diferiram, quando comparados à autoavaliação vocal e aos parâmetros perceptivos e acústicos. Isso pode ser explicado em função do curto tempo de intervenção, variável importante na verificação dos seus efeitos. Efeitos positivos foram significativamente relatados após a massagem vocal, concluindo-se que essa forma de tratamento pode ajudar os professores na manutenção do bem-estar vocal, durante a atividade profissional. Uma limitação desse estudo pode ser a ausência de grupo controle.

Foi possível observar, nesta revisão, que, em parte das pesquisas $^{(3,9,13,16)}$, incluíram-se apenas participantes do gênero feminino. Isso de deve ao fato de que, além das mulheres serem maioria na profissão docente, as professoras são conhecidas por apresentarem prevalência elevada de problemas vocais, devido à ocupação. $\mathrm{O}$ uso prolongado da voz é exigido nas atividades profissionais, muitas vezes em volume elevado e em ambientes acústicos desfavoráveis. $O$ efeito do gênero pôde ser observado, ainda, em pesquisas ${ }^{(3)}$ que mostraram que a estrutura da laringe e das pregas vocais podem também ser causas para a maior vulnerabilidade das mulheres aos problemas de voz e trauma nas pregas vocais. A adução insuficiente na parte dorsal da prega vocal e a forma curvada de sua borda aumentam o estresse mecânico no seu terço anterior, onde os nódulos podem aparecer. Na camada de superfície das pregas vocais das mulheres, foi observada menor quantidade de ácido hialurônico, em comparação com os homens, implicando em menor proteção contra traumas mecânicos nas pregas vocais.

A avaliação de sintomas vocais e da qualidade de vida relacionada à voz, também foi utilizada para comparar os resultados antes e após as intervenções vocais. Isso porque, por meio de questionários de autorrelato, é possível verificar o impacto da alteração na qualidade de vida de indivíduos com queixas vocais, de acordo com o gênero, faixa etária e uso vocal profissional ${ }^{(6)}$. Além disso, pode-se classificar os indivíduos como tendo vozes saudáveis ou disfônicas ${ }^{(19)}$. No entanto, para que o resultado dos efeitos das intervenções vocais seja fidedigno, é imprescindível a utilização combinada de questionários de autoavaliação vocal, da análise perceptivo-auditiva e de parâmetros acústicos. Alguns estudos ${ }^{(5,17)}$ observaram o aumento nos tempos máximos de fonação, porém, essa medida não pode ser considerada relevante no sentido clínico, pois não tem relação comprovada com distúrbios vocais ${ }^{(20)}$.

Em todos os estudos incluídos na presente revisão, os individuos participantes tiveram acompanhamento profissional, durante a execução dos exercícios. A experiência do profissional mostra-se como um fator importante na realização das intervenções ${ }^{(16)}$. Nesse sentido, existem falhas na literatura, em relação à efetividade do treinamento vocal na prevenção de distúrbios vocais em professores, especialmente no Brasil, onde grande número de pesquisas têm sido realizadas com essa população. Prevalecem, porém, estudos que enfatizam a avaliação vocal ${ }^{(10)}$.

Apesar de ser bem descrito na literatura o fato de os professores fazerem uso prolongado da voz e frequentemente a utilizarem em alta intensidade, em razão da acústica das salas de aula, não se tem ainda informações completas e seguras, oriundas de pesquisas realizadas em situações ideais, sobre quais são os métodos de intervenção mais adequados para esse público, como apontado na presente revisão. Foram encontrados poucos ensaios clínicos randomizados que abordassem esse tema, embora a estratégia de busca utilizada tenha sido ampla.

A partir dos estudos encontrados, concluiu-se que a intervenção combinada (direta e indireta) apresentou melhora significativa em parâmetros da qualidade vocal e na autoavaliação da voz. Nos estudos cujo foco era a comparação entre a intervenção combinada e intervenções isoladas (direta ou indireta), observou-se que a intervenção isolada não apresentou diferenças significativas, apesar de serem descritas melhoras em alguns parâmetros vocais avaliados. Em outro estudo(o), não foi verificada nenhuma evidência sobre a eficácia do treinamento direto ou indireto, ou uma combinação de ambos, para melhorar o desempenho vocal ou a autoavaliação da voz, quando comparado a nenhuma intervenção, evidenciando que são necessários estudos com metodologia adequada e medidas de resultados que reflitam melhor os objetivos das intervenções.

Em relação à qualidade dos estudos, foram utilizadas, como base, as normas consolidadas de Ensaios Reporting (CONSORT) para ensaios clínicos randomizados. Observou-se que a qualidade variou de fraca a muito boa, conforme observado na Tabela 2 . Esse dado aponta a necessidade de esforços para a produção de mais pesquisas em situações ideais. Dentre as limitações desta revisão, pode-se considerar a restrição quanto ao desenho metodológico dos estudos, incluindo apenas ensaios clínicos randomizados. Sugere-se que sejam realizadas outras revisões, incluindo outras classes de profissionais que necessitem de desempenho 
vocal efetivo durante o período de trabalho e que apresentem alta carga de uso vocal como, por exemplo, os teleoperadores ${ }^{(6,17)}$. Não foram encontradas outras revisões sistemáticas sobre intervenções vocais preventivas com professores, evidenciando também a falta de literatura nacional e internacional sobre o tema. Ainda, apesar do número restrito de artigos incluídos na presente revisão não permitir a generalização dos dados, observa-se a relevância dos resultados encontrados.

\section{CONCLUSÃO}

O presente estudo pôde esclarecer sobre os tipos de intervenção vocal utilizada com os professores e suas características principais. Observou-se que a abordagem combinada revelou resultados mais expressivos quando comparada com a intervenção isolada e foi possível observar, ainda, que a efetividade do treinamento vocal na prevenção de distúrbios vocais em professores não tem sido estudada amplamente.

A qualidade dos estudos incluídos variou de fraca a boa e foram encontradas algumas limitações, como a baixa produção de artigos na área, o tamanho amostral e o tempo de duração das intervenções. Nenhum dos trabalhos teve sua origem no Brasil, salientando a carência de ensaios clínicos controlados randomizados, com a perspectiva da prevenção para a população brasileira. Ressalta-se que esse fato pode ter se devido ao número reduzido de estudos nessa temática, produzidos em situação ideal de pesquisa, ou pela não indexação de algum estudo nas bases de dados pesquisadas. Destaca-se, portanto, a necessidade da realização de mais pesquisas em situações ideais, tanto nacionais, quanto internacionais, que abordem este tema, de modo a facilitar a escolha dos tipos de intervenção mais adequados para os profissionais que utilizam a voz como principal instrumento de trabalho.

\section{REFERÊNCIAS}

1. Henry MM, Johnson J, Foshea B. The effect of specific versus combined warm-up strategies on the voice. J Voice. 2009; 23(5):57276.

2. Hazlett DE, Duffy OM, Moorhead SA. Review of the impact of voice training on the vocal quality of professional voice users: implications for vocal health and recommendations for further research. J Voice. 2011;25(2):181-91.

3. Leppanen K, Ilomaki I, Laukkanen AM. One-year follow-up study of self-evaluated effects of voice massage, voice training, and voice hygiene lecture in female teachers. Logoped Phoniatr Vocol. 2010; 35(1):13-8.

4. Pasa G, Oates J, Dacakis G. The relative effectiveness of vocal hygiene training and vocal function exercises in preventing voice disorders in primary school teachers. Logoped Phoniatr Vocol. 2007;32(3):128-40.

5. Bovo R, Galceran M, Petruccelli J, Hatzopoulos S. Vocal problems among teachers: evaluation of a preventive voice program. J Voice.

\section{7;21(6):705-22}

6. Ruotsalainen JH, Sellman J, Lehto L, Jauhiainen M, Verbeek JH. . Interventions for preventing voice disorders in adults. Cochrane Database Syst Rev. 2007;17(4):CD006372.

7. Van Lierde KM, D’haeseleer E, Baudonck N, Claeys S, De Bodt M, Behlau M. The impact of vocal warm-up exercises on the objective vocal quality in female students training to be speech language pathologists. J Voice. 2011;25(3):115-21.

8. Milbrath RL, Solomon NP. Do vocal warm-up exercises alleviate vocal fatigue? J Speech Lang Hear Res. 2003;46(2):422-36.

9. Laukkanen AM, Leppänen K, Ilomäki I. Self-evaluation of voice as a treatment outcome measure. Folia Phoniatr Logop. 2009;61(1):5765 .

10. Dragone MLS, Ferreira LP, Giannini SPP, Zenari MS, Vieira VP, Behlau M. Voz do professor: uma revisão de 15 anos de contribuição fonoaudiológica. Rev Soc Bras Fonoaudiol. 2010;15(2):289-96.

11. Oxman A, Clarke M, editors. Cochrane reviewers' handbook 4.1.1: updated december 2000. In: Assessment of study quality. Oxford: The Cochrane Library; 2001.p.39-50.

12. Jadad AR, Moore RA, Carroll D, Jenkinson C, Reynolds DJ, Gavaghan DJ, et al. Assessing the quality of reports of randomized clinical trials: is blinding necessary? Control Clin Trials. 1996;17(1):1-12.

13. Duffy OM, Hazlett DE. The impact of preventive voice care programs for training teachers: a longitudinal study. J Voice. 2004;18(1):63-70.

14. Timmermans B, Coveliers Y, Meeus W, Vandenabeele F, Looy LV, Wuyts $\mathrm{F}$. The effect of a short voice training program in future teachers. J Voice. 2011;25(4):191-8.

15. Gillivan-Murphy P, Drinnan MJ, O'Dwyer TP, Ridha H, Carding P. The effectiveness of a voice treatment approach for teachers with self-reported voice problems. J Voice. 2006;20(3):423-31.

16. Leppanen K, Laukkanen AM, Ilomaki I, Vilkman E. A comparison of the effects of voice massage and voice hygiene lecture on self-reported vocal well-being and acoustic and perceptual speech parameters in female teachers. Folia Phoniatr Logop. 2009;61(4):227-38.

17. Ilomaki I, Laukkanen A M, Leppanen K, Vilkman E. Effects of voice training and voice hygiene education on acoustic and perceptual speech parameters and self-reported vocal well-being in female teachers. Logoped Phoniatr Vocol. 2008;33(2):83-92.

18. Wuyts FL, De Bodt MS, Molenberghs G, Remacle M, Heylen L, Millet B, et al. The dysphonia severity index: an objective measure of vocal quality based on a multiparameter approach. J Speech Lang Hear Res. 2000;43(3):796-809.

19. Behlau M, Oliveira G. Validation of self-assessment protocols in languages different from the original version. In: 27th World Congress of the International Association of Logopedics and Phoniatrics. Dinamarca; 2007.

20. Roy N, Merrill RM, Thibeault S, Gray SD, Smith EM. Voice disorders in teachers and the general population: effects on work performance, attendance, and future career choices. J Speech Lang Hear Res 2004;47(3):542-51. 